2011-9

\title{
Antiviral TRIMs: Friend or Foe in Autoimmune and Autoinflammatory Disease?
}

Caroline A. Jefferies

Royal College of Surgeons in Ireland

Claire Wynne

Technological University Dublin, claire.wynne@tudublin.ie

Rowan Higgs

Royal College of Surgeons in Ireland

Follow this and additional works at: https://arrow.tudublin.ie/scschbioart

Part of the Chemicals and Drugs Commons

\section{Recommended Citation}

Jefferies, C., Wynne, C, Higgs, R.: Antiviral TRIMs: friend or foe in autoimmune and autoinflammatory disease?. Nature Reviews, Immunology, 2011, Vol.11 (9), p. 617.25. doi: 10.1038/nri3043.

This Article is brought to you for free and open access by the School of Biological Sciences at ARROW@TU Dublin. It has been accepted for inclusion in Articles by an authorized administrator of ARROW@TU Dublin. For more information, please contact arrow.admin@tudublin.ie, aisling.coyne@tudublin.ie,gerard.connolly@tudublin.ie.

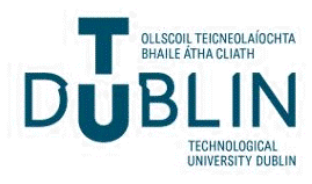


Anti-viral TRIMs -

Friend or Foe in autoimmune disease?

Claire Wynne ${ }^{1}$, Rowan Higgs ${ }^{2}$ and Caroline Jefferies ${ }^{1}$

${ }^{1}$ Molecular and Cellular Therapeutics, Royal College of Surgeons in Ireland, 123 St.

Stephen's Green, Dublin 2

${ }^{2}$ Immunology Research Centre, School of Biochemistry and Immunology, Trinity College Dublin, Dublin 2

Corresponding Author: Caroline Jefferies (cjefferies@rcsi.ie)

Ph. +353-1-4022226

Fax: $\quad+353-1-4025467$ 


\begin{abstract}
The concept that viral infection contributes to the development of autoimmune disease is supported by a wide range of clinical and experimental observations. TRIM family members, and particular the SPRY/PRY sub-class, are known for their involvement in anti-viral immunity - as either viral restriction factors or as regulators of pathways downstream of anti-viral RNA and DNA sensors. Interestingly, new data suggests that TRIMs also regulate the inflammasome. Therefore, whether as autoantigens or as regulators of anti-viral responses, evidence is emerging that TRIMs contribute to the development and pathology of autoimmune and autoinflammatory disease, making them novel targets for therapeutic manipulation.
\end{abstract}


The mammalian immune system has evolved to contain a wide range of membrane-bound and cytosolic pathogen recognition receptors (PRRs), which recognise and respond to specific molecular patterns from a variety of microbes. The intracellular signalling pathways that are activated downstream of PRRs drive the production of proinflammatory cytokines and type I interferons (IFNs), which initiate a rapid potent immune response and activate downstream effector and memory cells. Links exist between viral infections, induction of the innate immune response and the development of autoimmunity. For example, infection with viruses is often accompanied by the appearance of autoantibodies against self antigens. Epstein Barr Virus (EBV) infection for instance results in the generation of autoantibodies against autoantigens associated with the autoimmune disease systemic lupus erythrematosus (SLE), such as TRIM21/Ro52, Ro60 and La [1, 2]. These arise primarily as a result of molecular mimicry in the case of Ro60 and La, but also as a result of exposure of the autoantigens to the immune system following cellular insult. Ro52/TRIM21 for example, a cytosolic protein under normal conditions, is exposed at the cell membrane following ultraviolet irradiation of keratinocytes $[3,4]$. In addition, the pathogenesis of many autoimmune conditions is driven by the overproduction of cytokines associated with viral infection, such as tumour necrosis factor (TNF)- $\alpha$ and type I interferons (IFN). SLE patients characteristically display elevated type I IFN (IFN- $\alpha$ and IFN- $\beta$ ) levels in their serum during flare periods of this disease (reviewed in $[5,6])$. The molecular mechanism behind the overproduction of type I IFN is now well recognised to be as a result of recognition of self RNA and DNA complexes by pathogen recognition receptors (PRRs) such as the Toll-like receptor (TLR)- and -9, in addition to intracellular nucleic acid sensing systems.

In recent years, members of the tripartite motif (TRIM) family, perhaps best known as anti-viral proteins, have emerged as important regulators of PRR signalling pathways[7, 8]. Several TRIM proteins, such as TRIM5 $\alpha[9], 22[10]$ and 28[11], have direct anti-viral properties and together can restrict several stages of the viral replication cycle[7, 8, 12]. Complementing this, TRIMs have both positive and negative regulatory functions in PRR pathways[8]. Many TRIMS are both IFNinducible and regulate IFN production, which has led to the hypothesis that a subset of TRIMs, and in particular TRIM21[13], are involved in a negative regulatory feedback loop for IFN-mediated immunity[14]. The range of immune-related properties ascribed to the TRIM family continues to increase and recent research has implicated TRIM16 (also called estrogen-responsive B box protein)[15], TRIM20 (also called pyrin)[16] and TRIM30[17] in the regulation of interleukin (IL)-1 $\beta$ production via the inflammasome, which has a vital role in autoinflammatory conditions. Since IL-1 $\beta$ is well known to be involved in a multitude of inflammatory and autoimmune processes[18], an improved understanding of how TRIMs regulate inflammasome pathways might lead to the identification of therapeutic targets or the development of novel therapies for autoinflammatory disorders.

The unfolding story of the TRIMs is quickly establishing this family of proteins as crucial anti-viral and regulatory signalling molecules in PRR, inflammasome and autoimmune pathways. In this Review, we provide an update on the contribution of TRIMs to antiviral protection and innate immune regulation and discuss the 
emerging role of specific TRIMs in the molecular mechanisms and pathways associated with inflammation and autoimmunity.

\section{TRIMs - Structure, Function and Expression}

\section{Structure}

Since the first TRIM was cloned from Xenopus laevis mRNA in 1991[19], TRIM genes have been found in a wide range of species from fish[20] to pigs[21] to humans[22]. In total, genetic information is available for 68 human TRIMs (and several TRIM-like genes)[22], the majority of which have the characteristic really interesting new gene (RING)-finger, B-box and coiled coil (RBCC) domains (Figure 1 and Supplementary Figure 1). Most TRIM proteins share high sequence similarity in the RBCC domains. Indeed, the main variability is found in the $\mathrm{C}$-terminal domains, a region that appears to dictate the specific function of each individual protein (Box 1). Although there seems to be little correlation between genomic location and structure, six of the eight TRIM genes that cluster at chromosome 6p21-23 (TRIM10, 15, 26, 27, 38 and 39) and all of the TRIMs at 11p14-15 (TRIM5, 6, 21, 22, 34 and 68) contain a SPRY domain (Figure 1b), which is increasingly being linked to anti-viral and innate immune responses. It has been proposed that the human TRIM family is split into two distinct groups (Group 1 and 2) that evolve at different rates and are subject to different selective pressures[22]. Group 1 members are found in both vertebrate and invertebrate species and contain a range of C-terminal domains. By contrast, members of Group 2, which include the majority of the clustered genes at 6p21-23 and 11p14-15, are not found in invertebrates and contain a C-terminal SPRY domain. Overall, the evidence suggests that Group 2 TRIMs are the largest sub-group, (accounting for 40/71 family members (as shown in Supplementary Figure 1)) and may have a more recent origin, and thus have evolved more rapidly, than members of Group 1[22]. This accelerated divergence and positive selection of Group 2 TRIMs might, in part, be attributable to the critical role that many PRY/SPRY-containing TRIMs have in the immune response against rapidly mutating viruses as will be discussed below.

\section{E3 ligase activity}

The RING-finger domain of TRIMs and other protiens is long established as conferring the ability to act as an E3 ubiquitin ligase[23]. Following the contribution of E1 and E2 enzymes, this enables the RING-finger-containing protein to assist in the transfer of single or multiple ubiquitin moeities from the E2 ligase onto a target protein. As the majority of TRIMs possess a RING-finger domain, it is not surprising that many family members have been shown to self-ubiquitinate and/or to promote the ubiquitination of target proteins[24-26] including important proinflammatory and IFN signalling molecules, such as inhibitor of NFKB kinases (IKKs)[27] and IFN regulatory factors (IRFs)[28]. Ubiquitinated proteins are subsequently degraded, activated or trafficked to other locations in the cell - the particular outcome is thought to be mostly dependent on the specific lysine on the ubiquitin molecule 
employed to facilitate poly-ubiquitination[29]. TRIMs are also known to contribute to the post-translational modification of target proteins mediated by SUMO[30] and ISG15[31].

\section{Expression}

TRIMs are expressed in the cytosol and nucleus of most cell types, indicative of a family of proteins with a wide array of functions. The expression of TRIM19/PML is particularly distinct in that it forms the mass bulk of nuclear bodies, and has several physiological roles including the regulation of transcription[32] and interferonmediated innate immunity[33]. Many other TRIMs seem to localise to unidentified cellular compartments; an observation confirmed by studying the localization patterns of mutated TRIMs[34]. As many TRIMs have direct and indirect anti-viral properties, a reasonable hypothesis was that TRIMs would be induced following activation of the classic anti-viral type I IFN pathway. Accordingly, several studies have shown that various TRIMs are type I IFN-inducible (Table 1)[35-38].

On the basis of mRNA expression data of murine TRIMs in various immune cell subsets, Rajsbaum et al. noted that many TRIMs, including TRIM19, 20 and 21, were highly expressed in macrophages and DCs under conditions which are known to induce type I IFN (i.e. influenza virus infection or TLR-9 stimulation), suggesting that these mRNAs are IFN-inducible. Indeed, the observed induction of mRNA expression was abrogated in IFN- $\alpha /-\beta R^{-/-}$mice [37], confirming that these TRIMs are induced via a type I IFN-dependent mechanism.

In a separate study, human TRIM mRNAs were analysed for IFN-inducibility in several immune cell subsets [36]. Overall, 16 TRIM mRNAs were induced following type I IFN stimulation of primary monocyte-derived macrophages (MDM), while all but two were also upregulated in stimulated peripheral blood lymphocytes (PBL) (Table 1). Type II IFN stimulation induced the expression of only 7 TRIMs in each cell type. Both type I and II IFNs also downregulated a number of TRIMs in MDMs such as TRIM16, although only TRIM16L showed reduced mRNA expression in PBLs. In addition, FcyR signalling induced the mRNA expression of TRIM9 and 54 in MDM and downregulated 8 other TRIM mRNAs. Multiple TRIM mRNAs have also been shown to be either upregulated or downregulated following stimulation of human macrophages with LPS and IFN- $\gamma$ (Table 1). Overall, the cell-type specificity of TRIM expression, along with the selective inducibility following type I and II IFN signalling, allows the TRIM family to provide a multi-layered anti-viral response and simultaneously perform critical immunoregulatory functions.

\section{TRIMs, the anti-viral response and potential links with autoimmune and autoinflammatory disease}

The importance of TRIMs, particularly PRY/SPRY domain containing family members, in regulating the anti-viral response is well established and is underlined by the fact that many are targets for viral degradation [39, 40], and has been reviewed extensively $[7,8]$. Interestingly, a number of pathogens express pyrin-only domains 
which have been shown to inhibit IL1/IL18 production presumably by disrupting the inflammasome (reviewed in [41]). Conceivably, a similar strategy could be used by viruses to encode TRIM family proteins in order to limit the IFN or inflammatory response.

With respect to TRIMs in autoimmunity, the role for several members (TRIM21 and TRIM68 in particular) as autoantigens in autoimmune dieases such as SLE and Sjogren's syndrome (SS) is well established. However, given the link between antiviral responses and autoimmunity, newly emerging data on the role of this family as antiviral restriction factors and regulators of cytokine production downstream of viral recognition systems, suggests a role for TRIMs in protecting against autoimmune disease. In addition the growing realisation that certain TRIMs function to regulate the inflammasome, suggests a potential mechanism whereby defects in these proteins can contribute to autoinflammatory conditions associated with overproduction of IL-1 $\beta$. These various roles of TRIMs will now be explored, specifically in the context of implications for autoimmune and autoinflammatory conditions.

\section{TRIMs as autoantigens}

The protective antibody response accompanying viral infection is often accompanied by the appearance of autoantibodies against self proteins, with obvious pathogenic potential. Several mechanisms exist to explain how these autoantibodies arise. In general viral-induced production of autoantibodies against self proteins accompanies their exposure to the immune system as a result of cell death, changes in intracellular localisation or as a result of post-translational studies.

For example, recognition of TRIM21/Ro52, an autoantigen associated with SLE, is associated with altered membrane localisation and enhanced protein expression as a result of viral infection or cell injury [3, 4, 42]. TRIM68, an autoantigen in Sjogren's Syndrome and SLE, is also similarly exposed [43]. Several studies have reported the link between viral infection and the development of autoreactive antibodies and a number of these have demonstrated the production of antibodies specific to TRIM family members [2]. In addition to EBV infection giving rise to autoantibodies against Ro52/TRIM21, infection of mice with vaccinia virus or the non-cytopathic lymphocytic choriomeningitis virus revealed TRIM2-specific antibodies being induced. Whilst suggestive that TRIM2 expression or location within the cell maybe altered following viral infection, as yet no linkage for the involvement of TRIM2 in autoimmune disease exists. Recently autoantibodies against TRIM33 (TIF1 $\gamma$ ) and the intracellular apathogen recognition receptor (PRR) MDA-5 have been identified in patients suffering from the autoimmune connective tissue disease [44]. TRIM33 is best known for its role as a transcriptional repressor, although recently a role for it in regulating TGF- $\beta$ signalling has been described [45], indicating it may have an as yet undiscovered role in immune regulation. Whilst the pathophysiology of autoantibodies is clearly established, it is unclear as yet as to whether autoantibodies against TRIM family members have any specific role to play other than as markers of disease. The possibility exists that autoantibodies against specific 
targets may function to inappropriately sequester their targets, thus affecting their function. In the case of autoantibodies against Ro52/TRIM21, this may be particularly relevant, particularly in light of its role as a negative regulator of type I IFN production as discussed below.

\section{TRIMs as viral restriction factors and potential link with autoimmunity - an update}

With respect to a role for TRIMs as viral restriction factors there has been an explosion of information in recent years $[8,46,47]$. In particular, a study by Uchil et al [47] in which they screened various human and mouse TRIMs, demonstrated that TRIMs act nearly every stage in viral replication (summarised in table 2). This section aims to provide an update on emerging TRIMs in this area and potential links with autoimmunity.

TRIM5 $\alpha$ and TRIM22: Perhaps the best studied of all the TRIMs in this respect, TRIM5 $\alpha$ was discovered in 2004 as a retroviral restriction factor that determined the species restriction of HIV-1 [48] [49] [50]. It has multiple roles in inhibiting retroviral lifecycle, including recognition of the viral capsid by its SPRY/PRY domain [51] and more recently it has been shown to have direct effects on signal transduction pathways that may restrict retroviral replication [52], such as targeting NFKB and inflammasome activity as discussed below. Its close homologue, TRIM22, functions to inhibit HIV budding from infected cells [53]. Recent evidence also demonstrates an inverse correlation between viral load and expression of TRIM22, reinforcing its role as a potent restriction factor against HIV in vivo [54].

A genetic study investigating the role of human endogenous retroviruses (HERV) in multiple sclerosis (MS), demonstrated an inverse correlation between SNPs in TRIM5 $\alpha$ and MS. This suggests that certain SNPs may be protective against the development of MS, whether through restricting the effects of endogenous retroviral transmission or via its newly uncovered role as a regulator of NFKB/NLRP3 activation (see below). Indeed SNPs in TRIM5 $\alpha$ have already been demonstrated to be protective against HIV infection, revealing a protective role for this TRIM protein for human health.

TRIM28: Endogeneous retroviruses (ERV) are retroviral elements that comprise about $10 \%$ of mammalian DNA. They are transcriptionally silenced during early embryogenesis via histone and DNA methylation in order to protect genome integrity. TRIM28 has been identified as a key protein that supresses ERV expression via recruiting a complex series of proteins required to methylate histones [11, 55]. The recently emerging roles for human ERVs (HERV) in autoimmunity (ranging from direct effect of HERV genes on immune regulated pathways or HERV proteins acting as superantigens in the induction of autoantibody responses) and cancer suggest that TRIM28 (and potentially other TRIM family members) maybe important in regulating these diseases $[56,57]$

Therefore in addition to the role of TRIMs as viral restriction factors, it is now emerging that TRIMs play a more fundamental role in regulating anti-viral immune 
defences at various different levels as discussed below and combined these two important aspects of TRIM biology may explain the emerging data suggesting an involvement for TRIMs in autoimmune and autoinflammatory disorders.

\section{TRIMS and pathogen recognition pathways}

TRIM proteins act as either positive or negative regulators on the pathways downstream of pathogen recognition receptors, the majority requiring their E3 ligase activity for activation (via K63-linked polyubiquitination) or degradation (via K48linked polyubiquitination) of key signalling molecules. Anti-viral sensors principally detect viral nucleic acid, and under pathogenic circumstances can detect RNA and DNA released from damaged host cells. They include the Toll-like receptors (TLRs), the cytoplasmic RIG-like helicase receptors (RLRs), the inflammasome whose assembly is controlled by a subset of the NOD-like receptors (NLRs) and the newly described HIN family of proteins that function as intracellular double stranded (ds) DNA receptors. IFN- $\alpha$ and $-\beta$ (via activation of the transcription factors IRF3 and IRF7) and proinflammatory cytokines (via NFKB activation) are rapidly induced as a result of recruitment of specific adaptor and effector proteins as illustrated in Figure 2.

NFKB is the prototypical proinflammatory transcription factor, regulating the expression of proinflammtory cytokines (TNF, IL1, IL12 etc) and the upregulation of adhesion molecules and activation markers in order to promote the inflammatory response. Activation requires phosphorylation and degradation of the inhibitory protein IKB by upstream kinases IKK $\alpha$ and $\beta$, prior to release and nuclear translocation of NFKB. For both the TLR and RLR pathways the common convergence point in the activation of NFKB is the E3 ligase TRAF6. Autoubiquitination of TRAF6 results in recruitment of the adaptor proteins TAB2 and TAB3 and the kinase TAK1, resulting in activation of IKKs ([58] and reviewed in $[59,60])$. Highlighting the importance of the TAB1/TAK1 in regulating NFKB activation, TRIM5 $\alpha$ and its murine orthologue TRIM30 have been shown to interact with, ubiquitinate and degrade TAB2 and TAB3, resulting in disruption of the TRAF6/TAK1/TAB complex and inactivation of NFKB dependent pathways $([61,62])$.

The role of type I interferons in driving autoimmune disease is well established, with elevated IFN- $\alpha /-\beta$ observed in patients suffering from SLE, Sjogren's syndrome, dermatomyositis and psoriasis and is strongly correlated with both inflammation and disease-associated tissue damage. As IFN- $\alpha$ and $-\beta$ are produced in response to viral detection, either via their recognition by TLRs, RLRs or intracellular DNA sensors, it is components of these pathways that are generally targeted in order to limit or fine tune cytokine production. Activation of IRF3 or IRF7 downstream of TLR3, RLRs and cytosolic DNA receptors requires the activation of non-canonical IKK family members, TANK-binding kinase 1 (TBK1) and IKKE in a process involving TRAF3 and recruitment of signalling complexes to mitochondrial compartments. Activation of IRF7 downstream of TLR7 and TLR9 involves recruitment of IKK $\alpha$ to TRAF6-containing complexes at the endosome, in a process that requires ubiquitination in addition to phosphorylation of IRF7 [63]. As key regulators of anti-viral signalling, TRIM family 
members are increasingly recognised for their involvement in regulating these pathways via effects thus far identified at the level of the receptors themselves, the IKK complexes and as direct regulators of transcription factor activity or levels.

\section{Regulation of nucleic acid detection by TRIMs}

RIG-I like receptors (RLRs; including RIG-I itself, MDA-5 and Lgp2) and the AIM-2-like receptors (ALRs) recognise intracellular RNA and DNA, respectively. RIG-I encodes a $\mathrm{DExD} / \mathrm{H}$ box RNA helicase that contains a caspase recruitment domain and a helicase domain with intact ATPase activity, both of which are responsible for dsRNAmediated signalling. Mitochondrial antiviral signalling protein (MAVS), is an outermitochondrial membrane protein which functions downstream of RIG-I to mediate the activation of NFKB/IRF3, subsequent gene activation and type I IFN production in response to viral infection (PMID: [64]). MAVS contains an $\mathrm{N}$-terminal CARD-like domain and a C-terminal transmembrane domain, both of which are essential for MAVS signalling and subsequent interaction with TRAF3 in the case of interferonstimulated response element (ISRE) activation or TRAF6 in the case of NFKB activation. The human HIN-200 family of proteins include AIM2 and IFI16, both of which act as intracellular DNA receptors (reviewed in[65]). Whilst AIM-2 regulates inflammasome activity and hence IL-1 $\beta$ maturation [66], IFI16 is critical for sensing DNA viruses such as HSV-1 and EBV and drives the activation of IRF3 to enhance IFN$\beta$ production [67]. IFI16 does so via interacting with the ER-associated adaptor molecule STING (MITA, MYPS), promoting the relocalisation and activation of TBK1, resulting in IRF3 activation and IFN- $\beta$ production.

Regarding a role for TRIMs in regulating RLR and ALR activation, TRIM25 positively regulates IFN gene induction via K63-linked ubiquitination of RIG-I. In doing so ubiquitinatied RIG-I can now interact with the mitochondrial associated adaptor MAVS and recruit the downstream kinase TBK-1 and assemble the signalling complex required to initiate IRF3 and NFKB activation [68]. Interestingly, activation of RIG-I in this context has been shown to be dependent on recognition of linear ubiquitin chains formed by the newly identified linear ubiquitin assembly complex (LUBAC) [69]. However, a recent study suggests that the LUBAC complex degrades TRIM25 post stimulation in order to turn off and limit RIG-I activation [70]. Perhaps combined these studies indicate that sequential recruitment of RIG-I and TRIM25 to linear ubiquitin chains may function as a mechanism to regulate RIG-I induced IFN production. In another twist, caspase 12 has recently been shown to positively regulate RIG-I mediated IFN-b in response to West Nile virus infection, potentially via promoting the interaction between RIG-I and TRIM25, although the precise mechanism behind this is as yet unknown [71]. Given the role of IFNs in SLE, it remains to be seen whether TRIM25 levels or activity are altered in patients suffering this systemic autoimmune disease.

With respect to DNA sensors TRIM56 has recently been shown to interact with and ubiquitinate STING (also known as MITA/ERIS/MYPS), an ER-associated adaptor protein that recruits TBK1 for dsDNA induced activation of IRF3 and induction of IFN [72]. The ubiquitination of STING is essential to facilitate dimerisation of the adaptor prior to the recruitment of TBK-1, thus marking TRIM56 
as a positive facilitator of this pathway. IFI16 requires STING for its activity and is a well known autoantigen in systemic autoimmune disease [73]. The absolute requirement for STING in the production of IFN- $\beta$ has come from the recent report of a loss-of-function variant in an American cohort that correlates with an approximate $90 \%$ loss of IFN- $\beta$ inducibility in subjects homozygous for the mutation [74]. Interestingly, increased expression of murine HIN200 family members has been demonstrated to result in lupus-like syndrome in these mice, thus positively correlating expression of this family of proteins with autoimmunity (reviewed in [75, 76]. Whether or not TRIM56 plays a role in regulating autoimmune progression remains to be determined.

\section{Regulation of canonical and non-canonical IKK family members}

Given the absolute requirement for IKK involvement in activation of either NFKB or IRF3/7 downstream of TLRs, RLRs or ALRs, they are an ideal point at which to regulate flux through these pathways. Accordingly TRIMs have been shown to either positively or negatively regulate responses to viruses by targeting these key proteins.

Probably one of the earliest reports of a TRIM family member negatively regulating antiviral signalling pathways demonstrated that TRIM27/Ret Finger Protein (RFP) could interact with both IKK $\alpha /$ IKK $\beta$ and IKKE /TBK1 complexes and inhibit transcription of NFKB- and ISRE-dependent reporter genes [27]. Although no known role for TRIM27 in autoimmunity is described, a weak association between polymorphisms in TRIM27 and Crohn's disease, rheumatoid arthritis and type I diabetes has been suggested from network-based analysis of results from published Genome Wide Association Studies (GWAS) [77]. Recently a role for TRIM23 in regulating IKKs has been demonstrated. TRIM23 interacts with IKK $\gamma /$ NEMO and catalyses its ubiquitination via K27-linked polyubiquitin chains, a modification critically required for IRF3 and NFKB activation downstream of TLR3 and RIG-I [78].

Substantial evidence for cross talk between IKK family members exists, indicating the importance of regulatory proteins in fine tuning their activity [50]. Hence it is interesting to speculate that a stronger link between TRIM23 and TRIM27 in inflammatory and autoimmune disease may be uncovered in the future.

\section{Regulation of transcriptional responses}

Both GWAS studies and evidence from genetically modified mice have linked a number of transcription factors to the development of autoimmunity. Data is coming to light regarding the role for TRIMs in regulating transcription factor levels, DNA binding and localisation. Both TRIM19 and TRIM21 have been shown to regulate transcription factors directly, although the outcome of this activity (either positive or negative) varies greatly depending on the transcription factor involved.

TRIM19/PML (promyelomonocytic leukemia protein), the principle component of nuclear bodies, regulates a number of transcription factors, including STAT1 and NFkB, albeit with opposite effects. Regarding NFKB it has been demonstrated to inhibit its activity by binding to the RelA/p65 subunit and physically interfering with 
its binding to promoters $[79,80]$. The involvement of PML as a positive regulator of IFN- $\gamma$ signalling has been demonstrated to involve its RING domain, require SUMOylation of PML and result in enhanced phosphorylation of STAT1 [81]. In addition to affecting STAT1 activity, PML has also been shown to interact with PML Zinc Finger (PMLZF) protein and in doing so promote its binding to ISG elements and enhance IFN- $\gamma$ mediated anti-viral gene expression [33]. Interestingly, with respect to a role for PML in autoimmunity, autoantigens against PML itself and other nuclear body proteins have been detected in patients suffering from primary biliary cirrhosis, an autoimmune disease of the liver, indicating that these proteins are potentially overexpressed in this condition or become upregulated during cellular stress [82].

A number of independent studies have demonstrated that Ro52/TRIM21 interacts with and regulates members of the IRF family of transcription factors, and that certainly, in the case of IRF3 and IRF7, promotes their ubiqutination and subsequent degradation in order to limit type I IFN production downstream of pathogen recognition. Two groups independently knocked out Ro52/TRIM21 [13, 83]. In the study by Espinosa et al [13], the Ro52 $2^{-/}$mice develop a lupus-like syndrome as a result of activation of TLR7 and TLR9 and overproduction of type I IFNs and proinflammatory cytokines. However Yoshimi et al [83] failed to observe any obvious phenotype in their mice, although they did report impaired ubiquitination of IRF3 and IRF8 (another substrate for Ro52/TRIM21). Different targeting strategies or environments have been suggested to underpin the differences in phenotypes observed ([84]), although perhaps the explanation lies in the manner in which both groups of mice were challenged - the first group investigating anti-viral responses and the second group challenging the mice with the bacterial TLR- 4 agonist, LPS. Whatever the explanation, the results from Espinosa et al are intriguing, indicating perhaps that in addition to being a prevalent autoantigen in SLE, Ro52/TRIM21 may have direct effects in safeguarding against the onset of autoimmunity.

Additional targets for Ro52/TRIM21 have been reported - IRF8 for example is positively regulated by Ro52/TRIM21 ubiquitination, resulting in modest increases in IL8-dependent cytokine production [85]. In a separate study Ro52/TRIM21 has been demonstrated to stabilise IRF3 levels and therefore positively regulates IRF3 activity during antiviral responses [86]. Together the data suggests that Ro52/TRIM21 functions as a positive regulator during the initial response to a viral challenge, sustaining IRF3 levels, but post-viral challenge it switches (possibly through recruitment of additional factors) to down regulating IFN production via degradation of IRF3 and IRF7. Ro52 also negatively regulates NFKB activity, by targeting IKK- $\beta$ for monoubiquitination [87]. Indeed a recent study demonstrates that Ro52 acts as a guardian against overactivation of IKK- $\beta$ and hence NFKB, by preferentially recognising phosphorylated IKK- $\beta$ (induced by the HTLV-1 protein TAX), and targeting it to autophagosomes for degradation following ubiquitination [88]. Thus the evidence strongly suggests that Ro52 plays an important role in preventing overactivation of both proinflammatory and type I IFN responses in response to viral infection and that if inactivated may contribute to the overproduction of cytokines associated with autoimmune disorders such as SLE. Given the fact that Ro52 can bind the Fc portion of antibodies coating internalised viruses [89], this raises the 
intriguing possibility that immune complexes and autoantibodies against Ro52, if internalised may have a role in altering the function, levels or location of Ro52.

\section{Autoinflammatory diseases - Inflammasome regulation by TRIM family members}

The NOD-like receptors (NLR) are cytosolic sensors that mainly recognise a wide spectrum of danger- and pathogen-assoicated molecular patterns (DAMPs and PAMPs) such as ATP, DNA, RNA, flagellin and uric acid crystals and hence function in the regulation of inflammation and apoptotic responses. The NLR family is composed of 22 genes in human and 34 in mouse [90]. NLRP1, NLRP3 and NLRC4 are NLR family proteins involved in inflammasome assembly along with the IFI200 family member absent in melanoma 2 (AIM2). Assembly of the inflammasome involves one of either NLRP1, NLRP3, NLRC4 or AIM2 together with ASC, PYCARD and Caspase-1 (outlined in figure 3 ). Once caspase-1 activation is triggered, pro-inflammatory cytokines IL-1 $\beta$ and IL-18 are processed and released [18]. Excessive inflammasome activation can cause autoinflammatory diseases including Type 2 Diatetes, Crohns Disease, Gout and Familial Mediterranean Fever [91-93]. The role of TRIMs in regulating inflammasome activity has been largely underappreciated. However emerging evidence suggests a critical role for these proteins in regulating both inflammasome assembly and IL-1 $\beta$ secretion. Interestingly two of the TRIMs involved in regulating inflammasome activity lack the RING domain (TRIM16 and TRIM20) indicating, that unless they interact with other TRIM-domain containing family members, ubiquitination is not required for regulation.

Familial Mediterranean Fever (FMF) is an autosomal recessive disorder characterised by periodic recurrent bouts of fever and inflammation, representing a lack of regulatory control of inflammasome activity and hence IL-1 $\beta$ production. The gene responsible (MEFV) encodes TRIM20/pyrin. Some controversy surrounds the role of pyrin in regulating inflammasome activity, with groups reporting seemingly contradictory results. However, new evidence from studies of the interaction between pyrin and another substrate PSTPIP1 (Proline-serine-threonine phosphatase-interacting protein 1) provide a molecular explanation for the role of pyrin in regulating inflammasome activity. These studies revealed that pyrin exists as a homotrimer in unstimulated cells and that stimulation releases an autoinhibitory interaction between the $\mathrm{N}$ terminal SPRY domain of pyrin and its $\mathrm{B}$ box. This now frees the SPRY domain of pyrin to facilitate interaction with ASC and thus promote activation of the inflammasome [94]. These studies concur with the phenotype of mice expressing the pyrin domain alone which show enhanced IL-1 $\beta$ processing, whereas full length pyrin inhibits these responses. Thus previous in vitro studies reporting opposing effects of pyrin on inflammasome activation can be explained in light of these studies - full length pyrin by virtue of this autoinhibitory linkage with its $B$ box represses inflammasome activation until the appropriate signal is received to cause its release (or processing) in order to drive inflammasome activity. Equally, processing of pyrin is similarly required in order to activate NFkB. Chae et al [16] 
suggest that pyrin is required to be cleaved by caspase-I in order to release the pyrin $\mathrm{N}$ terminal domain which then interacts directly with the p65 subunit of NFkB thus promoting nuclear entry and potentiating NFKB activation. This study also linked this process with FMF by demonstrating that elevated levels of cleaved pyrin are observed in leukocytes derived from FMF patients compared with controls, indicating enhanced NFKB activity correlate positively with FMF.

Apart from its well characterised role in FMF, additional links for pyrin with autoimmunity exist. For example, mutations in PSTPIP1[95], that prevent interaction with pyrin, have been linked to the development of PAPA syndrome (Pyogenic Arthritis, Pyoderma gangrenosum, Acne), an autoinflammatory condition. [96]. In addition, the known mutations in pyrin (M694A and V726A) that cause FMF have recently been described as risk alleles for development and morbidity of multiple sclerosis (MS) [97]. Sepcifically these alleles are over-represented in MS cohorts and the frequency of MS in FMF patients is twice as high [98].

TRIM16 is an estrogen responsive gene also known as estrogen-responsive $B$ box protein (EBBP), initially described for its involvement in keratinocyte differentiation [99] and retinoid metabolism [100]. Recently a role for this protein in regulating secretion of the proinflammatory cytokine IL-1 $\beta$ has been reported [101]. TRIM16 interacts with the inflammasome components Caspase 1 and Nalp1, and enhances IL-1 $\beta$ secretion through an alternative secretion pathway. In keeping with a role for TRIM16 in innate immunity and autoimmune inflammatory disease a recent study has demonstrated that expression of TRIM16 correlates with severity of rheumatoid arthritis (RA). Rheumatoid arthritis is typically characterised by pronounced hyperplasia of the synovium giving rise to the RA pannus. This produces proinflammatory cytokines and proteases that aid with the invasive properties of this tissue, giving rise to joint destruction and abnormalities associated with RA. A recent study into candidate genes that confer invasive properties to synoviocytes demonstrated that enhanced TRIM16 expression is closely associated with increased invasiveness. Whether the correlation between TRIM16 expression and enhanced invasiveness is as a result of altered TRIM16 regulation of IL-1 $\beta$ secretion remains to be elucidated.

Recently TRIM16 has been shown to bind and degrade vimentin in tumours [102]. Vimentin is a member of the intermediate filament family of proteins and presence of autoantibodies that recognise a citrulinated from of vimentin are strongly associated with RA and are used as both a diagnostic and prognostic marker. Interestingly, vimentin has been reported to be released by activated macrophages in response to TNF- $\alpha$ stimulation and have bacteriacidal properties via the stimulation of ROS production, raising the possibility that vimentin acts as a danger signal or DAMP (danger associated molecular pattern) in certain environments [103]. One wonders therefore whether a role exists for TRIM16 in regulating either vimentin levels or vimentin secretion in the innate immune response, potentially contributing to the pathology associated with RA.

TRIM30 has recently emerged as a candidate protein important in regulating IL-1 $\beta$ production in the mouse in addition to its role as a regulator of NFKB activation[104]. 
Hu et al demonstrate that TRIM30 regulates the inflammasome by inhibiting NLRP3activity and hence IL-1 $\beta$ secretion via an unknown mechanism involving ROS production [17]. Interestingly, in a separate study TRIM30, along with TRIM12, have been identified as candidate genes for defective negative selection of autoreactive thymocytes during development in the NOD mice[105]. As the NOD mice display a defect in central tolerance this potentially indicates a global role for TRIM30 in regulating autoimmune disease. As the closest human orthologue to TRIM30 are TRIM5 and 6, it is interesting to speculate a general role for TRIM5/6 in regulating inflammatory cytokine production and hence autoimmune inflammatory disorders.

It is well recognised that whilst normal activation of the inflammasome contributes to host defence, overactivation contributes to the pathogenesis of a range of autoinflammatory disorders, including FMF and Muckle-Wells syndrome. More recently the NLRP3 inflammasome was recently shown to be very important in regulating IL-1 $\beta$ production in response to IAPP in type 2 diabetic mice, again underlining the importance of regulating IL1- $\beta$ production and the activity of NLRP3 in preventing disease progression [106]. This raises the potential that TRIM family members (specifically TRIM20, 16 and 30) may be important contributors to the pathogenesis of these diseases.

\section{Future perspectives}

It is becoming increasingly evident that the TRIM family of E3 ligases, and specifically the PRY/SPRY domain containing subset, have an ever-increasing role to play in regulating signal transduction pathways downstream of anti-viral DNA and RNA sensors. Given the role of these receptors in infection, autoimmune and autoinflammatory disease, it is imperative that we elucidate the exact contribution of TRIMs to these pathways and define their precise role as potential regulators of autoimmune progression. There is currently much interest in E3 ligases as therapeutic targets. As such, given their apparent specificity, drugs that regulate TRIMs should be very useful in infection and autoimmune pathology. There is therefore a need to understand in detail how the activity of these family members are regulated and whether structural motifs within their $C$ terminal domains will be amenable to rationale drug design in order to control their activity. 


\section{Figure Legends}

Figure 1: TRIM structure and genomic clusters. a) Generic structure of a TRIM family member, including N-terminal RING-finger, B-boxes and coiled-coil domain, and variable C-terminal domain. Sequence homology of all TRIMs and SPRY-containing TRIMs are shown below using a colour spectrum with a red colour indicating high sequence homology. Regions in each colour spectrum represent the homology of the amino acids encoding the domains directly above in the general TRIM structure. Alignments and the subsequent homology colour spectra were produced using TCoffee (http://www.ebi.ac.uk/Tools/msa/tcoffee/). b) TRIM loci on chromosome 6 and 11 . While the majority of TRIMs are scattered throughout the genome, two TRIM clusters are located on chromosome 6p21-23 and chromosome 11p14-15. These TRIMs share close evolutionary relationships suggestive of recent gene duplications, e.g. TRIM21/Ro52 and TRIM68. Most TRIMs located these clusters share a SPRY domain, known to be important for protein-ligand interactions in immune regulation. Phylogenetic and molecular evolutionary analyses were conducted using MEGA version 4 [107]. Abbreviations: ARF, ADP ribosylation factorlike; BR, bromodomain; COS, C-terminal subgroup one signature; FN3, fibronectin type 3; FIL, filamin-type immunoglobulin; MATH, meprin and tumour-necrosis factor receptor-associated factor homology; PHD, plant homeodomain; RING, really interesting new gene; TRIM, tripartite-motif; TM, transmembrane.

Supplementary Figure 1: TRIM family and domain structures. Domain structure was based on the SMART online domain prediction program (http://smart.emblheidelberg.de/). The coiled-coil domain was not detected using this program but is thought to be present in the majority of TRIMs.

Figure 2: TRIM family members have been associated with the regulation of proinflammatory cytokine and type I IFN production via TLR and RLR pathways.

TRIM5 $\alpha$ and its murine orthologue TRIM30 $\alpha$ have been shown to negatively regulate NFKB promoter activity via their degradation of TAB2/3 [104].Another direct negative regulator of this pathway is TRIM19. TRIM19 binds $p 65$ hence interfering with promoter binding [108]. Both TRIM27 and TRIM21 have been shown to interact with the IKKs repressing their activity and as a result pro-inflammatory cytokine production $[27,88]$. TRIM21 also interacts with the IRFs 3, 7,8, each interaction resulting in repression of NFKB and IFN promoter activity [85, 109-111]. On the contrary, some TRIMs function as positive regulators of these pathways. TRIM25 postively regulates IFN gene induction via its binding to the RIG-I receptor which in turn results in MAVS interaction [68]. TRIM56 can bind the ER-associated adaptor protein STING, which then recruits TBK1 for dsDNA induced activation of IRF3 and IFN induction [72]. TRIM23 has been demonstrated to interact with TRAF3/6 and NEMO downstream of TLR3/4 and RIG-I resulting in NFKB and IFN pathway activation [78]. TRIM20 can also positively impact NFKB promoter activity as a result of Caspase 1 cleavage with results in pyrin domain release and interaction with the p65 subunit [16]. 
Figure 3: TRIMs have been associated with the regulation of both NLRP1 and NLRP3 inflammasomes. Inflammasome activation results in the production of proinflammatory cytokines, namely IL-1 $\beta$ and IL-18. TRIM16 has been shown to interact with both Caspase 1 and NALP1 resulting in an enhancement in IL-1 $\beta$ secretion [101]. It has been suggested that murine TRIM30 $\alpha$ inhibits NLRP3 activity via an unknown mechanism involving ROS (reactive oxygen species) production [17]. Pyrin/TRIM20 exists as a homotrimer in unstimulated cells. Inflammatory stimuli results in the release of an autoinhibitory interaction between pyrins $\mathrm{N}$ terminal SPRY domain and its B box domain. Once activated, the SPRY domain then interacts with ASC (apoptosis-associated speck-like protein containing a caspase recruitment domain) promoting its oligomerisation and activation of the inflammasome [94, 112]. 


\section{Box 1. TRIM protein structure and function}

Members of the TRIM family are easily identifiable due to the presence of the characteristic RING-finger, B-box and coiled coil (RBCC) domains. These N-terminal domains, which form the tripartite motif, are highly conserved across the TRIM family. The RING finger is a zinc finger motif and has been widely shown to confer E3 ligase activity to many of the TRIM proteins ${ }^{[24-26]}$. The B-boxes, which are comprised of B-box 1 and/or 2, are zinc-binding domains, mutations in which are associated with diseases such as familial Mediterranean fever (and acute promyelocytic leukemia ${ }^{\mathrm{B} 1}$. The coiled coil domain is important for certain protein-protein interactions and can promote homo- or heterodimer formations, inducing the formation of complexes, such as TRIM19/PML nuclear bodies. While the preceding $\mathrm{N}$-terminal domains are highly conserved between the TRIMs, the majority of the diversity in TRIM proteins is found in the C-terminal region, which consists of at least one of 10 unique domains and forms the basis of a TRIM classification system ${ }^{\mathrm{B} 2}$. The most prevalent C-terminal motif is the SPRY domain, which when accompanied by a PRY sequence is also called a B30.2 domain and is known to facilitate protein-protein interactions $^{\mathrm{B} 3}$. Several TRIMs have been shown to bind target antibodies and proteins through the PRY/SPRY domain. Other TRIM C-terminal domains include COS, FN3, FIL, PHD, BR, MATH, ARF and TM (Figure 1), some of which have been shown to mediate both immune and non-immune functions. See Ozato et al. ${ }^{[7]}$ and Nisole et al. ${ }^{[12]}$ for a more detailed discussion of TRIM protein structure. Abbreviations: ARF, ADP ribosylation factor-like; $B R$, bromodomain; COS, C-terminal subgroup one signature; FN3, fibronectin type 3; FIL, filamin-type immunoglobulin; MATH, meprin and tumour-necrosis factor receptor-associated factor homology; PHD, plant homeodomain; PML, promyelocytic leukemia; RING, really interesting new gene; TRIM, tripartite-motif; TM, transmembrane.

\section{Box refs (not already in text):}

B1. Torok M, Etkin LD. Two B or not two B? Overview of the rapidly expanding B-box family of proteins. Differentiation. 2001 Mar;67(3):63-71.

B2. Short KM, Cox TC. Subclassification of the RBCC/TRIM superfamily reveals a novel motif necessary for microtubule binding. J Biol Chem. 2006 Mar 31;281(13):8970-80

B3. Rhodes DA, de Bono B, Trowsdale J. Relationship between SPRY and B30.2 protein domains. Evolution of a component of immune defence? Immunology. 2005 Dec;116(4):411-7. 


\section{References Body Text and Figure Legends}

1. Poole, B.D., et al., Lupus-like autoantibody development in rabbits and mice after immunization with EBNA-1 fragments. J Autoimmun, 2008. 31(4): p. 362-71.

2. McClain, M.T., et al., Early events in lupus humoral autoimmunity suggest initiation through molecular mimicry. Nat Med, 2005. 11(1): p. 85-9.

3. Casciola-Rosen, L.A., G. Anhalt, and A. Rosen, Autoantigens targeted in systemic lupus erythematosus are clustered in two populations of surface structures on apoptotic keratinocytes. J Exp Med, 1994. 179(4): p. 1317-30.

4. Ohlsson, M., R. Jonsson, and K.A. Brokstad, Subcellular redistribution and surface exposure of the Ro52, Ro60 and La48 autoantigens during apoptosis in human ductal epithelial cells: a possible mechanism in the pathogenesis of Sjogren's syndrome. Scand J Immunol, 2002. 56(5): p. 456-69.

5. Banchereau, J. and V. Pascual, Type I interferon in systemic lupus erythematosus and other autoimmune diseases. Immunity, 2006. 25(3): p. 383-92.

6. Ronnblom, L. and G.V. Alm, Systemic lupus erythematosus and the type I interferon system. Arthritis Res Ther, 2003. 5(2): p. 68-75.

7. Ozato, K., et al., TRIM family proteins and their emerging roles in innate immunity. Nat Rev Immunol, 2008. 8(11): p. 849-860.

8. McNab, F.W., et al., Tripartite-motif proteins and innate immune regulation. Current Opinion in Immunology, 2011. 23(1): p. 46-56.

9. Stremlau, M., et al., The cytoplasmic body component TRIM5[alpha] restricts HIV-1 infection in Old World monkeys. Nature, 2004. 427(6977): p. 848-853.

10. Gao, B., et al., Tripartite motif-containing 22 inhibits the activity of hepatitis $B$ virus core promoter, which is dependent on nuclear-located RING domain. Hepatology, 2009. 50(2): p. 424-433.

11. Rowe, H.M., et al., KAP1 controls endogenous retroviruses in embryonic stem cells. Nature, 2010. 463(7278): p. 237-240.

12. Nisole, S., J.P. Stoye, and A. Saib, TRIM family proteins: retroviral restriction and antiviral defence. Nat Rev Micro, 2005. 3(10): p. 799-808.

13. Espinosa, A., et al., Loss of the lupus autoantigen Ro52/Trim21 induces tissue inflammation and systemic autoimmunity by disregulating the IL-23â€"Th17 pathway. The Journal of Experimental Medicine, 2009. 206(8): p. 1661-1671.

14. Bolland, S. and A. Garcia-Sastre, Vicious circle: systemic autoreactivity in Ro52/TRIM21-deficient mice. The Journal of Experimental Medicine, 2009. 206(8): p. 1647-1651.

15. Munding, C., et al., The estrogen-responsive B box protein: a novel enhancer of interleukin-1 [beta] secretion. Cell Death Differ, 2006. 13(11): p. 19381949.

16. Chae, J.J., et al., The familial Mediterranean fever protein, pyrin, is cleaved by caspase- 1 and activates $N F-\kappa B$ through its $N$-terminal fragment. Blood, 2008. 112(5): p. 1794-1803.

17. Hu, Y., et al., Tripartite-Motif Protein 30 Negatively Regulates NLRP3 Inflammasome Activation by Modulating Reactive Oxygen Species Production. The Journal Of Immunology, 2010. 185(12): p. 7699-7705.

18. Shaw, P.J., M.F. McDermott, and T.-D. Kanneganti, Inflammasomes and autoimmunity. Trends in Molecular Medicine, 2011. 17(2): p. 57-64. 
19. Reddy, B.A., M. Kloc, and L. Etkin, The cloning and characterization of a maternally expressed novel zinc finger nuclear phosphoprotein (xnf7) in Xenopus laevis. Developmental Biology, 1991. 148(1): p. 107-116.

20. van der Aa, L., et al., A large new subset of TRIM genes highly diversified by duplication and positive selection in teleost fish. BMC Biology, 2009. 7(1): p. 7.

21. Ando, A., et al., Genomic sequence analysis of the 238-kb swine segment with a cluster of TRIM and olfactory receptor genes located, but with no class I genes, at the distal end of the SLA class I region. Immunogenetics, 2005. 57(11): p. 864-873.

22. Sardiello, M., et al., Genomic analysis of the TRIM family reveals two groups of genes with distinct evolutionary properties. BMC Evolutionary Biology, 2008. 8(1): p. 225.

23. Germana, M. and D.-R. Graciana, TRIM/RBCC, a novel class of lsquosingle protein RING fingerrsquo E3 ubiquitin ligases. BioEssays, 2005. 27(11): p. 1147-1157.

24. Wada, K. and T. Kamitani, Autoantigen Ro52 is an E3 ubiquitin ligase. Biochemical and Biophysical Research Communications, 2006. 339(1): p. 415-421.

25. Gack, M.U., et al., TRIM25 RING-finger E3 ubiquitin ligase is essential for RIG-I-mediated antiviral activity. Nature, 2007. 446(7138): p. 916-920.

26. Yamauchi, K., et al., Ubiquitination of E3 ubiquitin ligase TRIM5 $\alpha$ and its potential role. FEBS Journal, 2008. 275(7): p. 1540-1555.

27. Zha, J., et al., The Ret Finger Protein Inhibits Signaling Mediated by the Noncanonical and Canonical I $\kappa$ B Kinase Family Members. The Journal Of Immunology, 2006. 176(2): p. 1072-1080.

28. Higgs, R. and C.A. Jefferies, Targeting IRFs by ubiquitination: regulating antiviral responses. Biochemical Society Transactions, 2008. 036(3): p. 453458.

29. Weissman, A.M., Themes and variations on ubiquitylation. Nat Rev Mol Cell Biol, 2001. 2(3): p. 169-178.

30. Chu, Y. and X. Yang, SUMO E3 ligase activity of TRIM proteins. Oncogene, 2011. 30(9): p. 1108-1116.

31. Zou, W. and D.-E. Zhang, The Interferon-inducible Ubiquitin-protein Isopeptide Ligase (E3) EFP Also Functions as an ISG15 E3 Ligase. Journal of Biological Chemistry, 2006. 281(7): p. 3989-3994.

32. Zhong, S., P. Salomoni, and P.P. Pandolfi, The transcriptional role of PML and the nuclear body. Nat Cell Biol, 2000. 2(5): p. E85-E90.

33. Xu, D., et al., Promyelocytic Leukemia Zinc Finger Protein Regulates Interferon-Mediated Innate Immunity. Immunity, 2009. 30(6): p. 802-816.

34. Reymond, A., et al., The tripartite motif family identifies cell compartments. EMBO J, 2001. 20: p. 2140 - 2151.

35. Asaoka, K., et al., A retrovirus restriction factor TRIM5alpha is transcriptionally regulated by interferons. Biochem Biophys Res Commun, 2005. 338: p. 1950 - 1956.

36. Chelbi-Alix MK, P.L., Quignon F, Koken MH, Venturini L, Stadler M, Pavlovic J, Degos L, de Thé H., Induction of the PML protein by interferons in normal and APL cells. Leukemia, 1995. 12: p. 2027-33. 
37. Rajsbaum, R., J.P. Stoye, and A. O'Garra, Type I interferon-dependent and independent expression of tripartite motif proteins in immune cells. European Journal of Immunology, 2008. 38(3): p. 619-630.

38. Carthagena, L., et al., Human TRIM Gene Expression in Response to Interferons. PLoS ONE, 2009. 4(3): p. e4894.

39. Liu, X., et al., Enterovirus 71 induces degradation of TRIM38, a potential E3 ubiquitin ligase. Virol J. 8(1): p. 61.

40. Gack, M.U., et al., Influenza A virus NS1 targets the ubiquitin ligase TRIM25 to evade recognition by the host viral RNA sensor RIG-I. Cell Host Microbe, 2009. 5(5): p. 439-49.

41. Xiao, T.S., Subversion of innate immune signaling through molecular mimicry. J Clin Immunol. 30(5): p. 638-42.

42. Casciola-Rosen, L. and A. Rosen, Ultraviolet light-induced keratinocyte apoptosis: a potential mechanism for the induction of skin lesions and autoantibody production in LE. Lupus, 1997. 6(2): p. 175-80.

43. Billaut-Mulot, O., et al., SS-56, a novel cellular target of autoantibody responses in SjÃ Igren syndrome and systemic lupus erythematosus. The Journal of Clinical Investigation, 2001. 108(6): p. 861-869.

44. Hoshino, K., et al., Anti-MDA5 and anti-TIF $1-\gamma$ antibodies have clinical significance for patients with dermatomyositis. Rheumatology, 2010.

45. Randrianarison-Huetz, V., et al., Gfi-1B controls human erythroid and megakaryocytic differentiation by regulating TGF- $\beta$ signaling at the bipotent erythro-megakaryocytic progenitor stage. Blood, 2010. 115(14): p. 27842795.

46. Ozato, K., et al., TRIM family proteins and their emerging roles in innate immunity. Nat Rev Immunol, 2008. 8(11): p. 849-60.

47. Uchil, P.D., et al., TRIM E3 Ligases Interfere with Early and Late Stages of the Retroviral Life Cycle. PLoS Pathog, 2008. 4(2): p. e16.

48. Lee, K. and V.N. KewalRamani, In defense of the cell: TRIM5 $\alpha$ interception of mammalian retroviruses. Proceedings of the National Academy of Sciences of the United States of America, 2004. 101(29): p. 10496-10497.

49. Stremlau, M., et al., Species-Specific Variation in the B30.2(SPRY) Domain of TRIM5\{alpha\} Determines the Potency of Human Immunodeficiency Virus Restriction. J. Virol., 2005. 79(5): p. 3139-3145.

50. Stremlau, M., et al., The cytoplasmic body component TRIM5alpha restricts HIV-1 infection in Old World monkeys. Nature, 2004. 427: p. 848 - 853.

51. Sebastian, S. and J. Luban, TRIM5alpha selectively binds a restrictionsensitive retroviral capsid. Retrovirology, 2005. 2(1): p. 40.

52. Tareen, S.U., et al., An expanded clade of rodent Trim5 genes. Virology, 2009. 385(2): p. 473-483.

53. Barr, S.D., J.R. Smiley, and F.D. Bushman, The Interferon Response Inhibits HIV Particle Production by Induction of TRIM22. PLoS Pathog, 2008. 4(2): p. e1000007.

54. Singh, R., et al., Association of TRIM22 with the Type 1 Interferon Response and Viral Control during Primary HIV-1 Infection. J. Virol., 2011. 85(1): p. 208-216.

55. Matsui, T., et al., Proviral silencing in embryonic stem cells requires the histone methyltransferase ESET. Nature, 2010. 464(7290): p. 927-931. 
56. Balada, E., M. Vilardell-Tarrés, and J. Ordi-Ros, Implication of Human Endogenous Retroviruses in the Development of Autoimmune Diseases. International Reviews of Immunology, 2010. 29(4): p. 351-370.

57. Romanish, M.T., C.J. Cohen, and D.L. Mager, Potential mechanisms of endogenous retroviral-mediated genomic instability in human cancer.

Seminars in Cancer Biology, 2010. 20(4): p. 246-253.

58. Kanayama, A., et al., TAB2 and TAB3 activate the NF-kappaB pathway through binding to polyubiquitin chains. Mol Cell, 2004. 15(4): p. 535-48.

59. Landstrom, M., The TAK1-TRAF6 signalling pathway. Int J Biochem Cell Biol. 42(5): p. 585-9.

60. Carpenter, S. and L.A. O'Neill, Recent insights into the structure of Toll-like receptors and post-translational modifications of their associated signalling proteins. Biochem J, 2009. 422(1): p. 1-10.

61. Shi, M., et al., TRIM30 alpha negatively regulates TLR-mediated NF-kappa B activation by targeting TAB2 and TAB3 for degradation. Nat Immunol, 2008. 9(4): p. 369-77.

62. Tareen, S.U. and M. Emerman, Human Trim5alpha has additional activities that are uncoupled from retroviral capsid recognition. Virology. 409(1): p. 113-20.

63. Chariot, A., The NF-kappaB-independent functions of IKK subunits in immunity and cancer. Trends Cell Biol, 2009. 19(8): p. 404-13.

64. Seth, R.B., et al., Identification and characterization of MAVS, a mitochondrial antiviral signaling protein that activates NF-kappaB and IRF 3. Cell, 2005. 122(5): p. 669-82.

65. Mondini, M., et al., Role of the Interferon-Inducible Gene IFI16 in the Etiopathogenesis of Systemic Autoimmune Disorders. Annals of the New York Academy of Sciences, 2007. 1110(1): p. 47-56.

66. Hornung, V., et al., AIM2 recognizes cytosolic dsDNA and forms a caspase-1activating inflammasome with ASC. Nature, 2009. 458(7237): p. 514-8.

67. Unterholzner, L., et al., IFI16 is an innate immune sensor for intracellular DNA. Nat Immunol, 2010. 11(11): p. 997-1004.

68. Gack, M., et al., TRIM25 RING-finger E3 ubiquitin ligase is essential for RIGI-mediated antiviral activity. Nature, 2007. 446: p. 916 - 920.

69. Zeng, W., et al., Reconstitution of the RIG-I Pathway Reveals a Signaling Role of Unanchored Polyubiquitin Chains in Innate Immunity. Cell, 2010. 141(2): p. 315-330.

70. Inn, K.-S., et al., Linear Ubiquitin Assembly Complex Negatively Regulates RIG-I- and TRIM25-Mediated Type I Interferon Induction. Molecular Cell, 2011. 41(3): p. 354-365.

71. Wang, P., et al., Caspase-12 controls West Nile virus infection via the viral $R N A$ receptor RIG-I. Nat Immunol, 2010. 11(10): p. 912-919.

72. Tsuchida, T., et al., The Ubiquitin Ligase TRIM56 Regulates Innate Immune Responses to Intracellular Double-Stranded DNA. Immunity, 2010. 33(5): p. 765-776.

73. Mondini, M., et al., A novel autoantigen to differentiate limited cutaneous systemic sclerosis from diffuse cutaneous systemic sclerosis: The interferoninducible gene IFI16. Arthritis \& Rheumatism, 2006. 54(12): p. 3939-3944.

74. Jin, L., et al., Identification and characterization of a loss-of-function human MPYS variant. Genes Immun. 
75. Choubey, D., R. Deka, and S.M. Ho, Interferon-inducible IFI16 protein in human cancers and autoimmune diseases. Front Biosci, 2008. 13: p. 598-608.

76. Choubey, D. and R. Panchanathan, Interferon-inducible Ifi200-family genes in systemic lupus erythematosus. Immunol Lett, 2008. 119(1-2): p. 32-41.

77. Baranzini, S.E., et al., Pathway and network-based analysis of genome-wide association studies in multiple sclerosis. Hum Mol Genet, 2009. 18(11): p. 2078-90.

78. Arimoto, K.-i., et al., Polyubiquitin conjugation to NEMO by triparite motif protein 23 (TRIM23) is critical in antiviral defense. Proceedings of the National Academy of Sciences, 2010. 107(36): p. 15856-15861.

79. Wu, W.S., Z.X. Xu, and K.S. Chang, The promyelocytic leukemia protein represses A20-mediated transcription. J Biol Chem, 2002. 277(35): p. 317349.

80. Wu, W.S., et al., Promyelocytic leukemia protein sensitizes tumor necrosis factor alpha-induced apoptosis by inhibiting the NF-kappaB survival pathway. J Biol Chem, 2003. 278(14): p. 12294-304.

81. El Bougrini, J., L. Dianoux, and M.K. Chelbi-Alix, PML positively regulates interferon gamma signaling. Biochimie. 93(3): p. 389-98.

82. Stinton, L.M., et al., Autoantibodies to $G W$ bodies and other autoantigens in primary biliary cirrhosis. Clin Exp Immunol. 163(2): p. 147-56.

83. Yoshimi, R., et al., Gene disruption study reveals a nonredundant role for TRIM21/Ro52 in NF-kappaB-dependent cytokine expression in fibroblasts. $\mathrm{J}$ Immunol, 2009. 182(12): p. 7527-38.

84. Ozato, K., et al., Comment on "Gene disruption study reveals a nonredundant role for TRIM21/Ro52 in NF-kappa B-dependent cytokine expression in fibroblasts". J Immunol, 2009. 183(12): p. 7619; author reply 720-1.

85. Kong, H., et al., Cutting edge: autoantigen Ro52 is an interferon inducible E3 ligase that ubiquitinates IRF-8 and enhances cytokine expression in macrophages. J Immunol, 2007. 179: p. 26 - 30.

86. Yang, K., et al., TRIM21 is essential to sustain IFN regulatory factor 3 activation during antiviral response. J Immunol, 2009. 182(6): p. 3782-92.

87. Wada, K., et al., Ro52-mediated monoubiquitination of IKK\{beta\} downregulates NF-\{kappa\}B signaling. J Biochem, 2009.

88. Niida, M., M. Tanaka, and T. Kamitani, Downregulation of active IKK[beta] by Ro52-mediated autophagy. Molecular Immunology. In Press, Corrected Proof.

89. Mallery, D.L., et al., Antibodies mediate intracellular immunity through tripartite motif-containing 21 (TRIM21). Proceedings of the National Academy of Sciences, 2010. 107(46): p. 19985-19990.

90. Ting, J.P.Y., et al., The NLR Gene Family: A Standard Nomenclature. Immunity, 2008. 28(3): p. 285-287.

91. Kolly, L., et al., Expression and function of the NALP3 inflammasome in rheumatoid synovium. Immunology, 2010. 129(2): p. 178-185.

92. Meng, G., et al., A Mutation in the Nlrp3 Gene Causing Inflammasome Hyperactivation Potentiates Th17 Cell-Dominant Immune Responses. Immunity, 2009. 30(6): p. 860-874.

93. Dunne, A., Inflammasome activation: from inflammatory disease to infection. Biochem Soc Trans, 2011. 39(2): p. 669-73.

94. Waite, A.L., et al., Pyrin and ASC Co-Localize to Cellular Sites that Are Rich in Polymerizing Actin. Exp. Biol. Med., 2009. 234(1): p. 40-52. 
95. Shoham, N.G., et al., Pyrin binds the PSTPIP1/CD2BP1 protein, defining familial Mediterranean fever and PAPA syndrome as disorders in the same pathway. Proceedings of the National Academy of Sciences of the United States of America, 2003. 100(23): p. 13501-13506.

96. Yu, J.-W., et al., Pyrin Activates the ASC Pyroptosome in Response to Engagement by Autoinflammatory PSTPIP1 Mutants. Molecular Cell, 2007. 28(2): p. 214-227.

97. Unal, A., et al., Evaluation of common mutations in the Mediterranean fever gene in Multiple Sclerosis patients: Is it a susceptibility gene? Journal of the Neurological Sciences, 2010. 294(1-2): p. 38-42.

98. Yahalom G, K.S., Lidar M, Vaknin-Dembinsky A, Karussis D, Flechter S, Ben-Chetrit E, Livneh A, Familial Mediterranean fever (FMF) and multiple sclerosis: an association study in one of the world's largest FMF cohorts. Eur J Neurol., 2011. 10: p. 1468-1331.

99. Beer, H.-D., et al., The Estrogen-responsive B Box Protein. Journal of Biological Chemistry, 2002. 277(23): p. 20740-20749.

100. Cheung, B.B., et al., The Estrogen-responsive B Box Protein Is a Novel Regulator of the Retinoid Signal. Journal of Biological Chemistry, 2006. 281(26): p. 18246-18256.

101. Munding, C., et al., The estrogen-responsive B-box protein: a novel enhancer of interleukin-1beta secretion. Cell Death Differ, 2006. 13: p. 1938 - 1949.

102. Marshall, G.M., et al., TRIM16 acts as a tumour suppressor by inhibitory effects on cytoplasmic vimentin and nuclear E2F1 in neuroblastoma cells. Oncogene, 2010. 29(46): p. 6172-6183.

103. Mor-Vaknin, N., et al., Vimentin is secreted by activated macrophages. Nat Cell Biol, 2003. 5(1): p. 59-63.

104. Shi, M., et al., TRIM30[alpha] negatively regulates TLR-mediated NF[kappa] $B$ activation by targeting TAB2 and TAB3 for degradation. Nat Immunol, 2008. 9(4): p. 369-377.

105. Liston, A., et al., Impairment of organ-specific T cell negative selection by diabetes susceptibility genes: genomic analysis by $m R N A$ profiling. Genome Biology, 2007. 8(1): p. R12.

106. Masters, S.L., et al., Activation of the NLRP3 inflammasome by islet amyloid polypeptide provides a mechanism for enhanced IL-1 [beta] in type 2 diabetes. Nat Immunol, 2010. 11(10): p. 897-904.

107. Tamura, K., et al., MEGA4: Molecular Evolutionary Genetics Analysis (MEGA) Software Version 4.0. Molecular Biology and Evolution, 2007. 24(8): p. 1596-1599.

108. Wu, W.-S., et al., Promyelocytic Leukemia Protein Sensitizes Tumor Necrosis Factor $\alpha$-Induced Apoptosis by Inhibiting the NF- $\kappa B$ Survival Pathway. Journal of Biological Chemistry, 2003. 278(14): p. 12294-12304.

109. Higgs, R., et al., Self protection from anti-viral responses--Ro52 promotes degradation of the transcription factor IRF7 downstream of the viral Toll-Like receptors. PLoS ONE, 2010. 5(7): p. e11776 PLoS ONE.

110. Higgs, R., et al., The E3 Ubiquitin Ligase Ro52 Negatively Regulates IFN\{beta\} Production Post-Pathogen Recognition by Polyubiquitin-Mediated Degradation of IRF3. J Immunol, 2008. 181(3): p. 1780-1786.

111. Young, J.A., et al., Fas-associated Death Domain (FADD) and the E3 Ubiquitin-Protein Ligase TRIM21 Interact to Negatively Regulate Virus- 
induced Interferon Production. Journal of Biological Chemistry, 2011. 286(8): p. 6521-6531.

112. Sakuma, R., A.A. Mael, and Y. Ikeda, Alpha Interferon Enhances TRIM5\{alpha\}-Mediated Antiviral Activities in Human and Rhesus Monkey Cells. J. Virol., 2007. 81(18): p. 10201-10206. 
Fig 1

a

- Confers E3 ligase activity

Absent in TRIM14

$16,29,44,66,70$

- Replaced with Pyrin

domain in TRIM20
- Present as either B-box

1 and 2 or B-box 2 alone

- Possible role in viral

recognition

- Mutations associated

with several human

diseases
- Role in protein-

protein interactions
- Most variable region

- Determines function

- Predominant motif is a C-terminal SPRY domain

- Other domains include COS, FN3, FIL, PHD, BR,

MATH, ARF and TM (see Supplementary Figure 1)

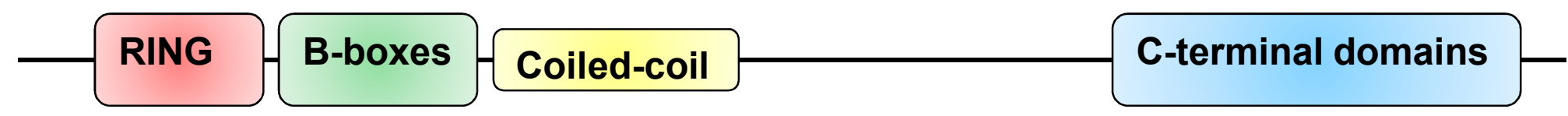

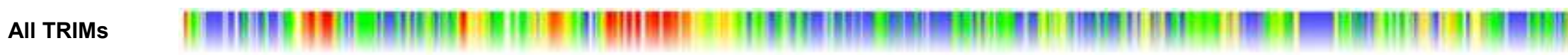

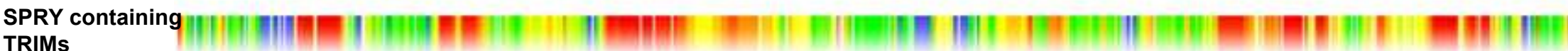

Homology Low $\rightarrow$ High

b
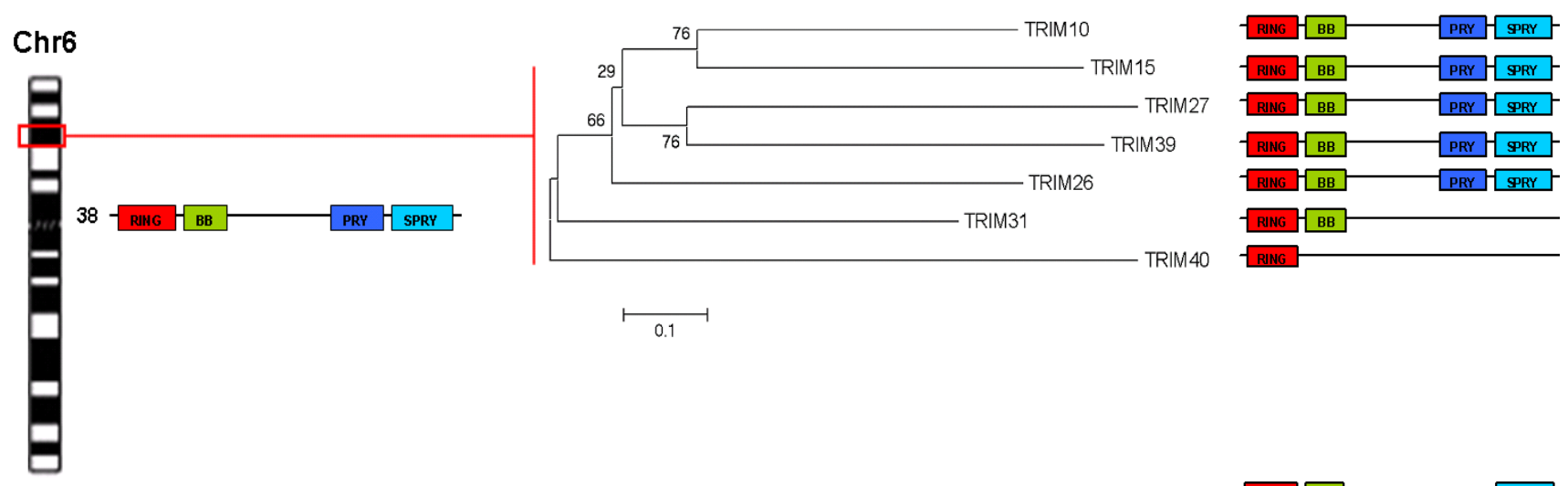

Chr11
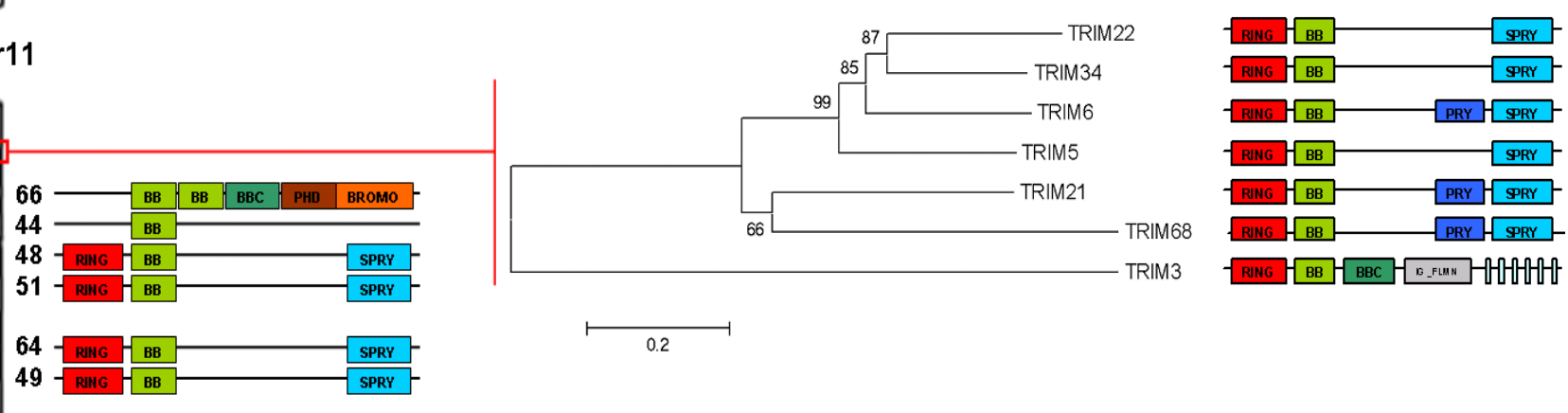

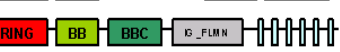


Supp

Fig 1
1 RING $\mathrm{BB}: \mathrm{BB}: \mathrm{BBC}$ FN3 $\mathrm{FPRY}$

2 RING BB BBC $16_{-}$FLMN

3 RING $\mathrm{BB}$ BBC 16 FLMN

$4-$ RING BB

5 RING $\mathrm{BB}$

6 RING BB

7 RING $B B$

$8-$ RING

$9-$ RING $\mathrm{BB}$ BB $\mathrm{BBC}$ FN3 $\mathrm{SPRY}$

$10-$ RING $\mathrm{BB}$ PRY SPRY

11 RING $\mathrm{BB}$ PRY $\mathrm{PPRY}$

13 RING $\mathrm{BB}$

14

$15-$ RING BB

16 BB $\mathrm{BB}$

17 RING BB

\begin{tabular}{ll|l|l|l|l|l|l|}
\hline 18 & RING & BB & BB & BBC & FN3 & PRY & SPRY \\
\hline
\end{tabular}

$19-$ RING BB BBZF

$20-$ PYR $B$ B

21 RING BB

22 RING $\mathrm{BB}$

23 - RING BB BB BBC

\begin{tabular}{l}
$24-\mathrm{RING}$ \\
\hline
\end{tabular}

25 RING PRY SPRY

$26-$ RING $B$ BB PRY SPRY

27 RING BB PRY SPRY

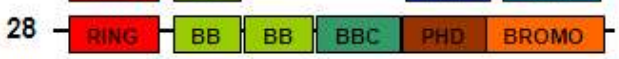

$29 \longrightarrow$ BB

$31-$ RING BB

32 RING BB

$33-$ RING

34 RING BB

$35-$ RING BB PRY SPRY

36 RING $\mathrm{BB}$

37 RING
38 RING $\mathrm{BB}$ PRY SPRY

$39-$ RING $\mathrm{BB}$

$40-$ RING

41 RING $\mathrm{BB}$

$42-$ RING BBZF

$43-$ RING BB

44 BB

$45-$ RING BB BB 16 FLMMN

$46-$ RING $\mathrm{BB}$

47 RING BB PRY

$48-$ RING BB

$49-$ RING $\mathrm{BB}$

$50-$ RING BB PRY SPRY

51 RING $\mathrm{BB}$

$52-$ RING BB

$54-$ RING BB

55 RING BB

$56-$ RING BB

58 RING BB

59 RING

$60-$ RING BB PRY SPRY

61 BING 62 BBZF

63 RING BB BBZF PRY SPRY

64 RING BB SPRY

65 RING BBZF SPRY

66 BB BB BBC PHD BROMO

$67-$ RING $\mathrm{BB}$ BB BBC $\mathrm{FN} 3 \mathrm{SPRY}$

68 RING $\mathrm{BB}$

$69-$ RING PRY SPRY

70 PRY SPRY

71 RING BB BB 16 ,FLM

$72-$ RING BB PRY SPRY

73 RING BB

74 RING BB
RING RING-finger domain

BB Bboxdomain

BBC Bbox C-terminal domain

FN3 Fibronectin type 3 domain

SPRY SPRY domain

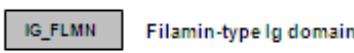

[ NHL repeat

PRY PRY clomain

TM Transmembrane domain

BBZF Bbox zinc finger domain

PYR PAAD/DAPIN/Pyrin domain

ARF ADP-ribosylation factor

PHD Plant homeodomain zinc finger domain

BROMO Bromodomain

MATH Meprin and TRAF domain 
Fig 2

TLR4

* Only found in mouse

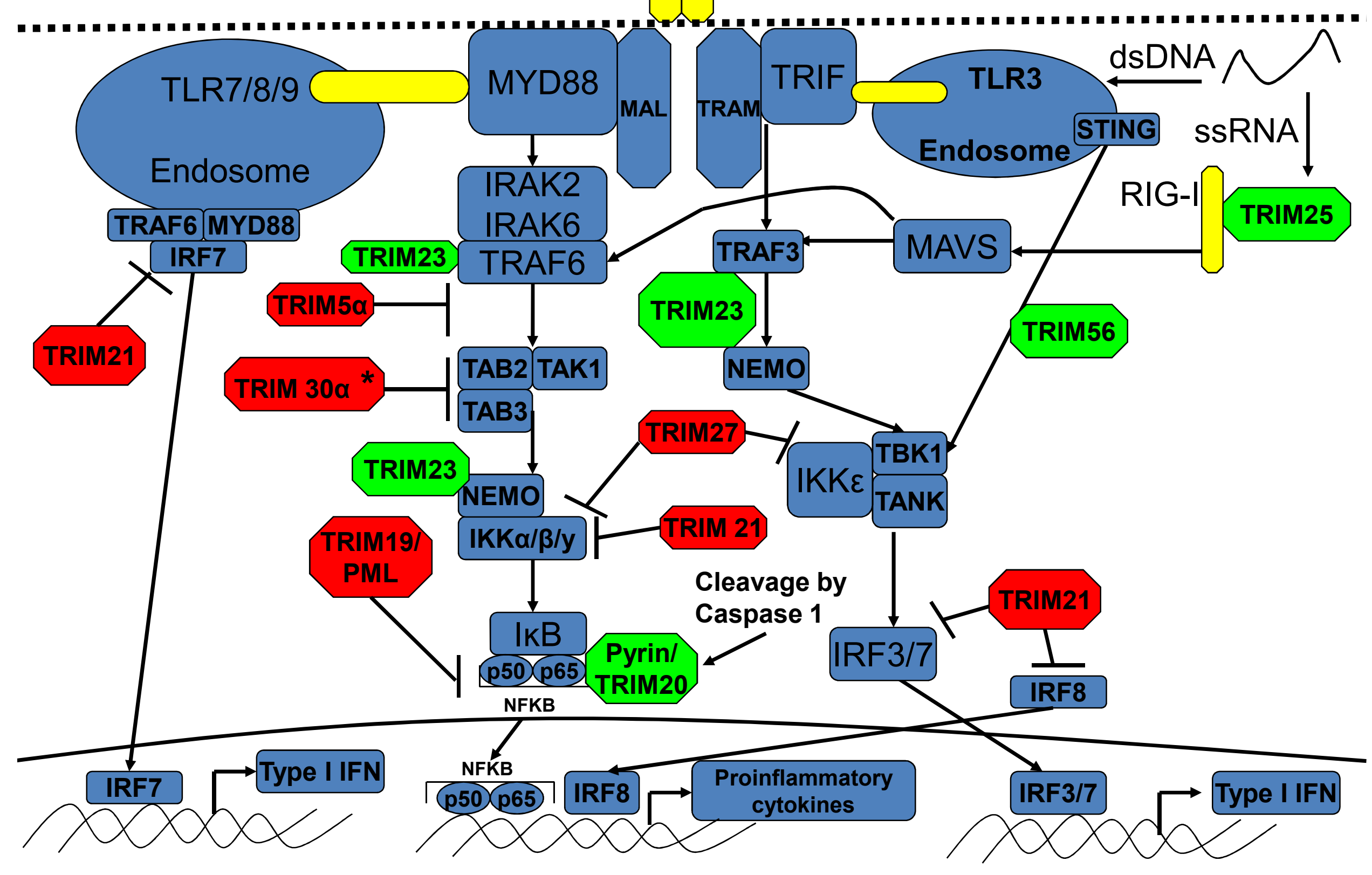


Fig 3

* Only found in mouse

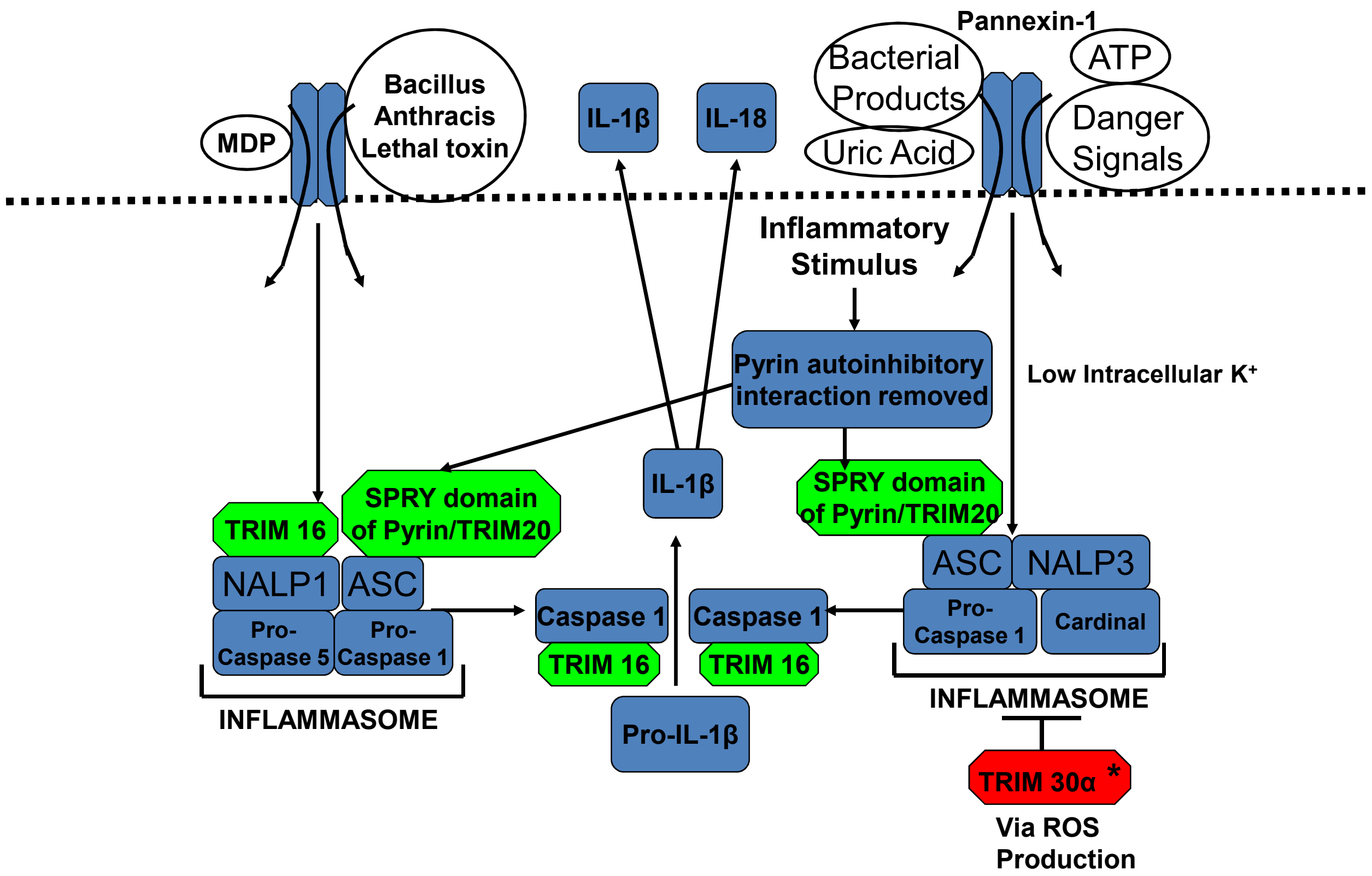




\begin{tabular}{|c|c|c|c|}
\hline \multicolumn{2}{|c|}{ Table 1: Human TRIM mRNA expression following immune cell stimulation } \\
\hline Stimulus & & Cell type & Study \\
\hline Induced & $38,56,58,69$ & & Carthagena et al. \\
\hline Type I IFN & $5,6,14,19,20,21,22,25,26,31,34,35$, & PBL, MDM & Carthagena et al. \\
\hline IFN $\gamma$ & $19,20,21,22,25,26,56,69$ & PBL, MDM & Martinez et al. \\
\hline LPS/IFN $\gamma$ & $\begin{array}{c}2,3,5,6,8,10,13,14,17,18,19,21,22, \\
25,26,29,31,33,34,35,38,45,46,48, \\
62,68\end{array}$ & Macrophage & \\
\hline IC & 9,54 & MDM & Carthagena et al. \\
\hline Repressed & $28,37,54,59,66$ & MDM & Carthagena et al. \\
\hline Type I IFN & $2,4,9,16,16 \mathrm{~L}, 28,32,37,54,59,66$ & MDM & Carthagena et al. \\
\hline IFN $\gamma$ & $16,23,27,32,44,52,66$ & Macrophage & Martinez et al. \\
\hline LPS/ IFN $\gamma$ & $22,32,34,44,47,58,59,66$ & MDM & Carthagena et al. \\
\hline IC & $\begin{array}{l}\text { Abbreviations: IC, immune complex; IFN, interferon; LPS, lipopolysaccharide; MDM, monocyte-derived } \\
\text { macrophage; PBL, peripheral blood lymphocyte. }\end{array}$ \\
\hline
\end{tabular}




\begin{tabular}{|c|c|c|c|c|c|}
\hline Effect of TRIM & HIV & N-MLV & B-MLV & ALV & Reference \\
\hline \multicolumn{6}{|l|}{ Viral entry } \\
\hline Enhance & 8 & 11 & - & ND & \\
\hline Decrease & $\begin{array}{l}\mathbf{5}, 11,26 \\
31\end{array}$ & $1,5,25,26,62$ & $25,26,62$ & ND & $\begin{array}{l}\text { Sebastian et al } \\
2005\end{array}$ \\
\hline \multicolumn{6}{|l|}{ Viral gene expression } \\
\hline Enhance & - & - & ND & - & \\
\hline Decrease & $\mathbf{5}, 11, \mathbf{2 2}$ & $\begin{array}{l}1,11,13,14 \\
21,27,31,32 \\
62\end{array}$ & ND & - & $\begin{array}{l}\text { Sakuma et al } 2007 \text {, } \\
\text { Tareen et al } 2009 \text {, } \\
\text { Singh et al } 2011\end{array}$ \\
\hline \multicolumn{6}{|l|}{ Viral release } \\
\hline Enhance & - & - & ND & - & \\
\hline Decrease & $\begin{array}{l}11,15,22 \\
26,32\end{array}$ & $\begin{array}{l}1,5,8,11,13 \\
14,15,19,21 \\
25,26,27,28 \\
31,32,35,62\end{array}$ & ND & 25,32 & Barr et al 2008 \\
\hline
\end{tabular}

